\title{
The unanticipated difficult airway - evolving strategies for successful salvage
}

\author{
Edward Crosby MD
}

A

CCORDING to Statistics Canada, just over a million persons aged $15 \mathrm{yr}$ and older had non-emergency surgery in 2001. Although not all of these patients necessarily had general anesthesia to permit surgery, this number would also be increased by those who underwent non-elective surgery, obstetrical anesthesia and persons younger than $15 \mathrm{yr}$ of age who underwent surgery. If we use the million cases as our starting point and accept that the incidence of the cannot intubate, cannot ventilate (CICV) scenario actually approximates the reported incidence of 0.01 to $0.07 \%$, there will be between 100 and 700 annual CICV events which must be managed across the country. Similarly, using often-quoted incidences for difficult intubation $(0.5-2 \%)$ there may be 5,000 to 20,000 difficult intubation events and for failed intubation $(0.045-0.3 \%)$, there may be 450 to 3,000 events. Despite careful preoperative evaluation, difficulties will not be predicted in many instances and strategies to manage the unanticipated difficult airway need to be pre-formulated and practiced.

Strategies for salvage of these clinical events were outlined by the American Society of Anesthesiologists (ASA) Task Force on Management of the Difficult Airway in 1993 and these were updated in 2003. ${ }^{1}$ The Canadian Airway Focus Group (CAFG) published recommendations for management of the unanticipated difficult airway in 1998 and the UK's Difficult Airway Society (DAS) published guidelines with a similar emphasis (unanticipated difficulties) in 2004.,.$^{2,3}$ A common theme in all of these publications is the advocacy for having a practiced strategy to facilitate the management of the difficult airway; all noted the value of alternatives to the direct laryngoscope when salvaging difficult airway scenarios. However, it is not clear how much influence these publications have had on current anesthesia airway management. Rosenstock and colleagues reported that $97 \%$ of anesthesia residents with an average of 60 months experience could not recall the ASA difficult airway algorithm; $17 \%$ of these had previously been involved in emergency situations with severe complications (death or brain damage) due to insufficient airway management. ${ }^{4}$

Tracheal intubation is still predominantly achieved with the use of direct laryngoscopy. Difficulties related to airway management largely involve failure to achieve tracheal intubation due to difficult direct laryngoscopy. A number of innovative new tools for tracheal intubation have been presented in recent years which address many of the factors which give rise to difficulties during direct laryngoscopy. ${ }^{2}$ Despite the apparent value of these techniques, anesthesia teachers seem to have been reluctant to teach them and practitioners slow to adopt them.

Rose and Cohen compared the techniques utilized and the airway management outcomes for 53,009 patients who had general anesthesia at their institution from 1991 to $1996.5,6$ The proportion of patients who underwent three or more direct laryngoscopies to achieve tracheal intubation were unchanged over time at $1.6 \%$ to $1.8 \%$. Failed intubation decreased over time but did not decrease below an incidence of $2.5 \%$ and the use of alternative techniques other than the fibreoptic bronchoscope was not increased over time; there was only a small increase in the use of the bronchoscope. Difficult laryngoscopy was most often managed with persistent attempts at direct laryngoscopy and the use of alternative approaches to tracheal intubation was uncommon (1.9\% of all tracheal intubations). In patients managed in this fashion (multiple direct laryngoscopy, rare use of alternate technology), there was a higher incidence of desaturation at induction,

From the Department of Anesthesiology, University of Ottawa, Ottawa Hospital, Ottawa, Ontario, Canada.

Address correspondence to: Dr. Edward Crosby, Department of Anesthesiology, University of Ottawa, Suite 2600, Tower 3, Ottawa Hospital - General Campus, 501 Smyth Road, Ottawa, Ontario K1H 8L6, Canada. Phone: 613-737-8187; Fax: 613-737-8189; E-mail: ecrosby@sympatico.ca 
esophageal intubation, dental damage and unexpected intensive care unit admissions. Mort also assessed the airway and hemodynamic complications associated with repeated attempts to perform tracheal intubation using direct laryngoscopy in 2,833 critically-ill patients. ${ }^{7} \mathrm{He}$ concluded that an increased number of attempts $(>2)$ was associated with a higher incidence of adverse events. The following complications were more common when patients were subjected to more than two direct laryngoscopic attempts compared to one or two attempts: severe hypoxemia (14x); esophageal intubation $(6 \times)$; regurgitation $(7 \times)$; aspiration $(4 \times)$; bradycardia $(4 \times)$; and cardiac arrest $(7 x)$.

It is likely that such over-reliance on direct laryngoscopy is a risk-enhancing behaviour that predisposes patients to morbidity and mortality. There is evidence that such behaviour is common among anesthesiologists. Rosenblatt surveyed a random sample of the active membership of the ASA. ${ }^{8}$ Physicians were asked to identify their preferred management technique for difficult airway scenarios involving cooperative adult patients who required tracheal intubation. Experienced practitioners tended to use higher risk induction techniques, used the direct laryngoscope almost exclusively, and their use of alternative devices and adjuncts was uncommon, occurring in less than $5 \%$ in all scenarios. Jenkins surveyed 833 Canadian anesthesiologists in the year 2000 to assess difficult airway management, training and access to airway equipment. ${ }^{9}$ The direct laryngoscope was still the preferred technology overall with the fibreoptic bronchoscope being the second most commonly used device. More experienced, male gender and older practitioners were again more likely to choose asleep induction for high-risk scenarios. Although respondents were asked to indicate their choice of technology to manage difficult airway scenarios, they were not asked to indicate their experience with the chosen technology. Kristensen similarly assessed airway management behaviour, experience and knowledge among Danish anesthesiologists and observed that both highrisk attitudes and behaviours were common. ${ }^{10}$ Again, there was little experience with or enthusiasm for using alternatives to the direct laryngoscope. Ezri's more recent survey of American anesthesiologists suggests that there may be an increasing willingness to use alternatives to the direct laryngoscope. ${ }^{11}$ However Ezri also observed that willingness persisted even when the anesthesiologists acknowledged that they were not comfortable nor experienced with the alternate technology that they proposed using in these difficult situations.

Although studies have demonstrated the individual utility of some new airway devices, there was until recently little published evidence that they have been incorporated widely into clinical practice or that their use had changed the outcomes in difficult airway events. Hung was among the first to observe and report that early conversion to an alternate technique when direct laryngoscopy was difficult, in that case the lighted stylet, resulted in successful tracheal intubation, performed quickly and without complications. ${ }^{12}$ More recently, Heidegger reported on a protocol for management of both anticipated and unanticipated difficult intubations which emphasized defaulting to the fibreoptic bronchoscope early when difficult laryngoscopy was anticipated or observed. ${ }^{13}$ Applied in 13,248 intubations, the protocol failed in only six patients $(0.045 \%)$ and again this strategy was associated with minimal morbidity. Finally, Combes reported on the efficacy of an institutional protocol employing the intubating laryngeal mask and gum elastic bougie. ${ }^{14}$ One hundred cases of unanticipated difficulties arose among 11,257 tracheal intubations. There were three deviations from the protocol and two patients were wakened without further airway management. All patients managed by the protocol were successfully ventilated and intubated.

In this issue of the Journal, Cook and colleagues report on the use of the ProSeal ${ }^{\mathrm{TM}}$ laryngeal mask airway (LMA) as a rescue device for failed intubation during rapid sequence induction of anesthesia and Burkle et al. report on the routine use of the fibreoptic bronchoscope for salvage of failed direct laryngoscopy. ${ }^{15,16}$ The value of Cook's contribution is the identification of the advantages of the ProSeal ${ }^{\mathrm{TM}}$ LMA compared to the LMA Classic ${ }^{\mathrm{TM}}$ for airway salvage in the setting of the full stomach and failed intubation. ${ }^{15}$ Burkle's paper is important as it identifies the role and value of an institutional pattern of practice in salvaging difficult direct laryngoscopy. ${ }^{16}$ Burkle reports on the common use of the bronchoscope after failed intubation with the direct laryngoscope and reports that in 93\% of the cases that it was employed, there was a successful resolution and a low incidence of morbidity. The difference between the experiences reported by Heidegger and Burkle was that the former derived a protocol mandating the use of the bronchoscope when direct laryngoscopy was difficult; the latter commonly defaulted to it as an institutional pattern of practice. In both instances, it was a highly successful salvage intervention when used, but in the case of Burkle, many patients who experienced failed intubation with the laryngoscope were not managed with a bronchoscope; its use was habitual but not protocol. It is acknowledged that $96 \%$ of the patients in Burkle's series who failed direct laryngoscopy were successfully intubated with adjuncts and alternatives to the laryngoscope even if a bronchoscope was not employed. 
Conventional airway practice employing a direct laryngoscope serves the majority of patients presenting for anesthetic care well. However, there is a population of patients for whom exclusive reliance on direct laryngoscopy is a high-risk strategy resulting in morbidity and occasional mortality. Unfortunately, there are difficulties encountered in accurately identifying these patients on an individual basis before induction of anesthesia and in many instances, difficulties will then be unanticipated. Although new evaluation techniques may reduce the incidence of unanticipated difficulties, these unanticipated events will continue to be a reality of clinical practice. Application of the guidelines and recommendations made by the ASA, the CAFG and now the DAS can result in the creation of local protocols which will likely prove to be highly effective in resolving airway crises resulting from difficult laryngoscopy. Integral to these protocols will be the application of adjuncts and alternatives to the direct laryngoscope; these devices seem capable of resolving many of these scenarios efficiently and safely. The emerging evidence suggests that experience with a small number of alternatives is sufficient for the practitioner to successfully resolve most difficulties. Thus, we should emphasize practitioners developing experience and a high comfort level with a limited number of alternatives rather than limited facility with a large range of alternatives. Opportunities for training with these newer technologies in both academic programs and in continuing education must be fostered. Although efforts are clearly underway at present to resolve some of these barriers to safe airway care, real airway safety will require further expenditure of both energy and resources. It is likely that motivation among anesthesiologists to improve care will be the factor that determines whether this enterprise is successfully concluded.

\section{L'intubation difficile imprévue - évolution des stratégies d'un sauvetage réussi}

Selon Statistique Canada, plus d'un million de personnes de 15 ans et plus ont subi une intervention chirurgicale non urgente en 2001. Tous ces patients n'ont pas nécessairement reçu une anesthésie générale et on pour- rait ajouter à leur nombre ceux qui ont subi une intervention non réglée ou une anesthésie obstétricale, et les opérés de moins de 15 ans. Le million de cas étant notre point de départ et l'incidence d'un scénario d'intubation impossible et de ventilation impossible (IIVI) étant d'environ 0,01 à $0,07 \%$, il y a de 100 à 700 incidents d'IIVI annuels à traiter à travers le pays. De même, selon les incidences souvent citées pour l'intubation difficile $(0,5-2 \%)$, il y aurait de 5000 à $20000 \mathrm{cas}$, et pour les échecs à l'intubation $(0,045-0,3 \%)$, il y aurait de 450 à 3000 incidents. Dans de nombreuses situations, les difficultés ne peuvent être prévues malgré une évaluation préopératoire minutieuse. La démarche à adopter devant une intubation difficile imprévue doit donc être préformulée et maîtrisée.

Des stratégies de sauvetage de ces incidents cliniques ont été esquissées par l'American Society of Anesthesiologists (ASA) Task Force on Management of the Difficult Airway en 1993 et mises à jour en 2003. ${ }^{1}$ Le Canadian Airway Focus Group (CAFG) a publié en 1998 des recommandations à appliquer en cas d'intubation difficile imprévue et l'UK's Difficult Airway Society (DAS) a publié des lignes directrices en insistant sur ce même problème en 2004..$^{2,3}$ Toutes ces publications plaident en faveur de la pratique de stratégies pour faciliter le contrôle des voies aériennes ; dans tous ces documents, on note la valeur de techniques de remplacement de la laryngoscopie directe dans des scénarios de sauvetage d'une intubation difficile. Toutefois, on ne sait pas vraiment si ces publications ont eu une influence importante sur la pratique courante de l'intubation en anesthésie. Rosenstock et ses collègues ont rapporté que $97 \%$ des résidents en anesthésie, dont l'expérience moyenne était de 60 mois, ne pouvaient se rappeler l'algorithme de l'intubation difficile de l'ASA ; $17 \%$ d'entre eux avaient été confrontés à des situations d'urgence aux complications sévères (mort ou dommage cérébral) en raison d'une protection inadéquate des voies aériennes. ${ }^{4}$

La laryngoscopie directe est toujours la technique prédominante pour l'intubation endotrachéale. Les difficultés de contrôle des voies aériennes relèvent en grande partie de l'échec de l'intubation endotrachéale lié à des problèmes de laryngoscopie directe. Ces dernières années, un certain nombre de nouveaux instruments novateurs ont été introduits pour l'intubation endotrachéale. Ils permettent de contrer de nombreuses difficultés de laryngoscopie directe. ${ }^{2}$ Malgré leur valeur apparente, les professeurs d'anesthésie semblent réticents à enseigner ces techniques et les praticiens tardent à les adopter.

Rose et Cohen ont comparé les techniques utilisées et la qualité du contrôle des voies aériennes chez 
53009 patients qui ont eu une anesthésie générale à leur hôpital entre 1991 et $1996 .^{5,6}$ La proportion de patients qui ont subi trois laryngoscopies directes ou plus pour l'intubation endotrachéale était stable dans le temps, soit de 1,6\% à 1,8\%. L'échec de l'intubation a diminué avec le temps, mais son incidence n'a pas baissé sous les 2,5\% et l'usage de techniques de remplacement, excluant le fibroscope bronchique, n'a pas augmenté ; il n'y a eu qu'une faible augmentation de l'usage du bronchoscope. Pour régler les difficultés de laryngoscopie, on a eu recours surtout à des essais répétés de laryngoscopie directe plutôt qu'à d'autres techniques d'intubation endotrachéale, lesquelles étaient rares ( $1,9 \%$ de toutes les intubations endotrachéales). Chez les patients intubés ainsi (laryngoscopies directes répétées, usage rare de technologie différente), il y a eu une forte incidence de désaturation à l'induction de l'anesthésie, d'intubation œsophagienne, de lésion dentaire et d'admissions imprévues à l'unité des soins intensifs. Mort et coll. ont aussi évalué les complications touchant les voies aériennes et l'hémodynamique à la suite d'essais répétés d'intubation endotrachéale par laryngoscopie directe chez 2833 patients gravement malades. ${ }^{7}$ Il a noté qu'un nombre accru d'essais $(>2)$ était associé à un plus grand nombre d'événements indésirables. Certaines complications étaient plus fréquentes avec plus de deux laryngoscopies directes comparées à une ou deux : hypoxémie sévère $(14 \times)$; intubation œsophagienne $(6 \times)$; régurgitation $(7 x)$; aspiration $(4 \times)$; bradycardie $(4 \times)$ et arrêt cardiaque $(7 \times)$.

Il est probable que ce recours fréquent à la laryngoscopie directe soit un comportement à risque exposant les patients à la morbidité et à la mortalité. Or, c'est une attitude manifestement fréquente chez les anesthésiologistes. Rosenblatt a mené un sondage auprès d'un échantillon aléatoire de membres actifs de l'ASA. ${ }^{8}$ Il a demandé aux médecins de désigner la technique qu'ils préfèrent utiliser dans les cas de difficulté d'intubation chez des patients adultes coopératifs qui nécessitent une intubation endotrachéale. Les médecins expérimentés avaient tendance à utiliser des techniques d'induction à plus haut risque, se servaient presque exclusivement de la laryngoscopie directe et l'usage d'autres instruments ou accessoires était rare, dans moins de $5 \%$ de tous les scénarios. Jenkins a sondé 833 anesthésiologistes canadiens en 2000 pour évaluer le contrôle des voies aériennes, la formation et l'accès au matériel d'intubation. ${ }^{9}$ La laryngoscopie directe était encore la technologie généralement utilisée, la fibroscopie bronchique étant le plus souvent la deuxième. Les praticiens de sexe masculin, plus expérimentés et plus âgés avaient plus tendance à réaliser l'induction chez un patient endormi dans les cas de scénarios à haut risque. On demandait aux répondants d'indiquer leur technologie de choix dans les cas d'intubation difficile, mais on ne leur demandait pas d'indiquer leur expérience avec cette technologie. Kristensen a aussi évalué le comportement, l'expérience et les connaissances des anesthésiologistes danois concernant le contrôle des voies aériennes et il a observé que les procédés et les comportements à haut risque étaient courants. ${ }^{10}$ Encore une fois, il y avait peu d'expérience avec des techniques de remplacement de la laryngoscopie directe ou peu d'enthousiasme à les utiliser. La plus récente enquête de Ezri auprès des anesthésiologistes américains montre qu'il y a un plus grand empressement à utiliser d'autres techniques que la laryngoscopie directe. ${ }^{11}$ Cependant Ezri a aussi observé que cette volonté a persisté même quand les anesthésiologistes ont reconnu qu'ils ne se sentaient pas à l'aise ni expérimentés avec la technologie de remplacement qu'ils proposaient pour les situations difficiles.

Des études ont démontré l'utilité particulière de nouveaux instruments d'intubation, mais il n'y a eu jusqu'à tout récemment que peu de publication prouvant que leur usage serait largement intégré à la pratique clinique ou qu'il ait modifié les événements entourant l'intubation difficile. Hung a été parmi les premiers à observer et à rapporter qu'une conversion précoce à une technique de remplacement en cas de laryngoscopie directe difficile, en l'occurrence le stylet lumineux, a permis une intubation endotrachéale réussie, rapide et sans complications. ${ }^{12}$ Plus récemment, Heidegger a présenté un protocole de traitement des intubations difficiles, prévues et imprévues, qui insistait sur la conversion précoce au fibroscope bronchique devant des difficultés laryngoscopiques prévues ou observées. ${ }^{13}$ Appliqué dans 13248 intubations, le protocole a échoué chez six patients seulement $(0,045 \%)$ et n'a été associé qu'à une morbidité minimale. Finalement, Combes a signalé l'efficacité d'un protocole institutionnel employant un masque laryngé d'intubation et une bougie d'intubation. ${ }^{14}$ Cent cas de difficultés imprévues sont ressortis parmi les 11257 intubations endotrachéales. Trois dérogeaient au protocole et deux patients ont été réveillés sans tentative supplémentaire d'accès aux voies aériennes. Tous les patients traités selon le protocole ont été ventilés et intubés avec succès.

Dans le présent numéro du Journal, Cook et ses collègues signalent l'usage du masque laryngé (ML) ProSeal ${ }^{\mathrm{TM}}$ comme dispositif de secours en cas d'échec de l'intubation pendant l'induction de l'anesthésie en séquence rapide et Burkle et coll. rapportent l'emploi 
régulier du fibroscope bronchique quand la laryngoscopie directe n'a pas réussi. ${ }^{15,16} \mathrm{La}$ valeur de l'article de Cook tient à la présentation des avantages du ML ProSeal $^{\mathrm{TM}}$ comparé au ML Classic $^{\mathrm{TM}}$ pour la protection des voies aériennes dans le contexte d'un estomac plein et d'un échec de l'intubation. ${ }^{15}$ L'article de Burkle est important, car il décrit le rôle et la valeur d'un modèle institutionnel de pratique de sauvetage de la laryngoscopie directe difficile. ${ }^{16}$ Burkle rapporte l'usage courant du bronchoscope après l'échec de l'intubation avec le laryngoscope direct et signale que dans $93 \%$ des cas où il est utilisé, le résultat est positif et l'incidence de morbidité est faible. La différence entre les expériences de Heidegger et Burkle est que le premier a construit un protocole recommandant l'usage du bronchoscope quand la laryngoscopie directe était difficile ; le second le choisit comme modèle courant de pratique institutionnelle. Ce sont deux interventions de sauvetage hautement réussies, mais dans le cas de Burkle, le bronchoscope n'a pas été utilisé dans de nombreux cas d'intubation impossible avec le laryngoscope ; son usage était fréquent, mais non intégré à un protocole. On admet que $96 \%$ des patients de la série de Burkle chez qui la laryngoscopie directe avait échoué ont été intubés avec succès avec des instruments d'appoint et des variantes du laryngoscope même si le bronchoscope n'a pas été employé.

L'intubation traditionnelle avec un laryngoscope direct répond bien aux besoins de la majorité des patients qui doivent être anesthésiés. Cependant, il y a une population de patients pour qui le recours exclusif à la laryngoscopie directe est une stratégie à haut risque entrânant morbidité et, parfois, mortalité. Malheureusement, il est difficile de dépister correctement ces patients sur une base individuelle avant l'induction de l'anesthésie et dans de nombreux cas, les problèmes seront donc imprévus. Même si de nouvelles techniques d'évaluation peuvent réduire la fréquence de difficultés imprévues, ces incidents vont continuer de se produire en clinique. L'application des lignes directrices et des recommandations faites par l'ASA, le CAFG et maintenant le DAS pourrait amener la création de protocoles locaux hautement efficaces à résoudre les crises autour de l'intubation résultant d'une laryngoscopie difficile. L'application d'instruments d'appoint et de variantes du laryngoscope direct devra faire partie de ces protocoles; ces instruments semblent capables de résoudre efficacement et en toute sécurité les nombreuses difficultés évoquées. Les dernières constatations permettent de penser que l'expérience acquise avec un petit nombre de techniques de remplacement est suffisante pour que le praticien soit en mesure de résoudre la plupart des difficultés. Nous devons donc insister sur le développement de l'expérience et d'un haut niveau de satisfaction avec un nombre limité de techniques de remplacement plutôt que d'une facilité limitée avec un grand nombre d'entre elles. Les occasions de formation avec ces nouvelles technologies doivent être encouragées autant par des programmes académiques que par une formation continue. Même si on fait déjà beaucoup d'efforts pour éliminer certaines barrières à l'apprentissage du contrôle efficace des voies aériennes, une intubation sécuritaire exigera d'autres dépenses en énergie et en ressources. C'est très certainement la motivation des anesthésiologistes pour l'amélioration des soins qui sera le facteur déterminant du succès ou de l'échec de l'entreprise.

\section{References}

1 American Society of Anesthesiologists Task Force on Management of the Difficult Airway. Practice guidelines for management of the difficult airway: an updated report by the American Society of Anesthesiologists Task Force on Management of the Difficult Airway. Anesthesiology 2003; 98: 1269-77.

2 Crosby ET, Cooper RM, Douglas MJ, et al. The unanticipated difficult airway with recommendations for management. Can J Anaesth 1998; 45: 757-76.

3 Henderson JJ, Popat MT, Latto IP, Pearce AC. Difficult airway society guidelines for management of the unanticipated difficult intubation. Anaesthesia 2004; 59: 675-94.

4 Rosenstock C, Østergaard D, Kristensen MS, Lippert A, Rubnau B, Rasmussen LS. Residents lack knowledge and practical skills in handling the difficult airway. Acta Anaesthesiol Scand 2004; 48: 1014-8.

5 Rose DK, Coben MM. The airway: problems and predictions in 18,500 patients. Can J Anaesth 1994; 41: 372-83.

6 Rose DK, Cohen MM. The incidence of airway problems depends on the definition used. Can J Anaesth 1996; 43: 30-4.

7 Mort TC. Emergency tracheal intubation: complications associated with repeated laryngoscopic attempts. Anesth Analg 2004; 99: 607-13.

8 Rosenblatt WH, Wagner PJ, Ovassapian A, Kain ZN. Practice patterns in managing the difficult airway by anesthesiologists in the United States. Anesth Analg 1998; 87: 153-7.

9 Jenkins K, Wong DT, Correa R. Management choices for the difficult airway by anesthesiologists in Canada. Can J Anesth 2002; 49: 850-6.

10 Kristensen MS, Møller J. Airway management behaviour, experience and knowledge among Danish anaesthesiologists - room for improvement. Acta 
Anaesthesiol Scand 2001; 45: 1181-5.

11 Ezri T, Szmuk P, Warters RD, Katz J, Hagberg CA. Difficult airway management practice patterns among anesthesiologists practicing in the United States: have we made any progress? J Clin Anesth 2003; 15: 418-22.

12 Hung OR, Pytka S, Morris I, Murphy M, Stewart RD. Lightwand intubation: II. Clinical trial of a new lightwand for tracheal intubation in patients with difficult airways. Can J Anaesth 1995; 42: 826-30.

13 Heidegger T, Gerig HJ, Ulrich B, Kreienbühl G. Validation of a simple algorithm for tracheal intubation: daily practice is the key to success in emergencies - an analysis of 13,248 intubations. Anesth Analg 2001; 92: 517-22.

14 Combes X, Le Roux B, Suen P, et al. Unanticipated difficult airway in anesthetized patients. Prospective validation of a management algorithm. Anesthesiology 2004; 100: 1146-50.

15 Cook TM, Brooks TS, Van der Westhuizen J, Clarke M. The ProSeal ${ }^{\mathrm{TM}}$ LMA is a useful rescue device during failed rapid sequence intubation: two additional cases. Can J Anesth 2005; 52: 630-33.

16 Burkle CM, Walsh MT, Harrison BA, Curry TB, Rose $S H$. Airway management after failure to intubate by direct laryngoscopy: outcomes in a large teaching hospital. Can J Anesth 2005; 52: 634-40. 